\title{
Lumen
}

Selected Proceedings from the Canadian Society for Eighteenth-Century Studies

\section{Temps historique et temps des oeuvres, propositions et réflexions}

\section{Jean M. Goulemot}

Volume 18, 1999

Representations of Time in the XVIIIth Century

Le temps et ses représentations au dix-huitième siècle

URI : https://id.erudit.org/iderudit/1012368ar

DOI : https://doi.org/10.7202/1012368ar

Aller au sommaire du numéro

Éditeur(s)

Canadian Society for Eighteenth-Century Studies / Société canadienne d'étude du dix-huitième siècle

ISSN

1209-3696 (imprimé)

1927-8284 (numérique)

Découvrir la revue

Citer cet article

Goulemot, J. M. (1999). Temps historique et temps des oeuvres, propositions et réflexions. Lumen, 18, 83-93. https://doi.org/10.7202/1012368ar

Copyright @ Canadian Society for Eighteenth-Century Studies / Sociéte canadienne d'étude du dix-huitième siècle, 1999
Ce document est protégé par la loi sur le droit d'auteur. L'utilisation des services d'Érudit (y compris la reproduction) est assujettie à sa politique d'utilisation que vous pouvez consulter en ligne.

https://apropos.erudit.org/fr/usagers/politique-dutilisation/ 


\section{Temps historique et temps des œuvres, propositions et réflexions}

Mon propos, ici, n'est pas de résumer mes travaux sur les représentations du devenir historique à l'âge classique. Je voudrais plus hardiment, plus imprudemment peut-être, proposer une réflexion et une programmatique, l'esquisse d'un chantier, à peine ouvert, plus rêvé qu'arpenté, mais dont je veux croire qu'il ouvre de nouvelles pistes de recherche et de nouveaux modes d'interrogation et de lecture des œuvres du XVIIIe siècle. L'interrogation, qui est ici la nôtre, sur les représentations du temps au XVIIIe siècle me suggère quelques remarques, que je vais d'entrée livrer.

Première constatation: le temps n'est pas un objet privilégié du questionnement des Lumières. On s'interroge peu individuellement sur le temps qui passe, la pauvreté du lyrisme en fournit la preuve, et peu même sur le vieillissement, si ce n'est par des voies obliques, comme Diderot quand il découvre son portrait par Van Loo ou constate qu'avec l'âge, selon cette belle formule, 'le gros bagage s'en ira. Je vois ces préparatifs du grand voyage se faire sans m'en soucier beaucoup' (lettre à Denise Diderot du 25 novembre 1778). Pour Jean-Jacques Rousseau, ce sont les arbres dénudés par l'automne vus des hauteurs de Menilmontant, le dégoût qu'éprouvent pour ses rides les très jeunes enfants, qui le poussent à s'interroger sur son âge et le temps qui passe (deuxième et huitième promenades). Il en naît un ressassement, angoissé et tendu, sur la vieillesse d'un homme au seuil de la mort. On objectera à l'affirmation de ce manque l'importance de la thématique des ruines dans les Salons de Diderot, Les saisons de Saint Lambert, qui sont l'occasion d'une réflexion sur le temps comme usure, temps individuel et temps collectif réunis. Mais il faut rappeler que ce sont là des chemins de traverse pour prendre en compte le temps historique et se souvenir que les civilisations sont mortelles, et qu'il faut oublier le bel optimisme qu'on prête, peutêtre un peu vite, à Voltaire. Ainsi il n'est pas insignifiant que Volney publie Les ruines en 1791 . Tout ceci est vrai. N'est-on pas alors dans les Lumières finissantes et les nouvelles sensibilités ne sont-elles pas en passe de triompher avec le néo-rousseauisme? En réalité, pour réelle 
qu'elle soit, la présence du temps ne revêt jamais aucun des caractères obsessionnels de la lyrique classique, médiévale ou renaissante et ne relève pas des angoisses de la finitude des formes politiques qui hante l'âge classique. Le procès de laïcisation sociale, qui est à l'œuvre au XVIIIe siècle, évacue l'interrogation religieuse sur le temps humain sans que parvienne à se constituer une conscience psychologique ou même morale du temps qui passe.

On se situe en fait dans un entre-deux difficile à estimer. Quelques références aux Salons, à la conscience de la vieillesse chez Diderot, Rousseau ou Voltaire montrent que, sans être un thème majeur, la réflexion sur le temps demeure bien présente dans l'espace intellectuel et affectif des Lumières. Ainsi, la naissance de l'autobiographie est non seulement un fait d'écriture, une prise de conscience de l'individuation psychologique, sentimentale et morale du moi, mais une illustration d'un rôle du temps dans le processus de formation et de transformation du moi profond. Ce n'est pas là la seule trace de cette présence du temps, de la durée, du processus de maturation qu'on puisse repérer dans les pratiques littéraires et les thématiques que met en œuvre le XVIIIe siècle.

Deuxième constatation: si le temps n'est pas un sujet essentiel du questionnement des Lumières, tout en jouant pourtant le rôle d'une présence secrète mais effective, il est vrai aussi que l'Histoire, le récit historique occupent une place fondamentale dans la réflexion philosophique. $\mathrm{Ce}$ double constat revient à dire peut-être qu'on ne parle guère du temps temps vécu, temps expérimenté, valeur du temps religieux ou laïque mais qu'on a transféré ce type de questions dans la pratique historique elle-même. On peut donc, dans cette perspective, légitimement s'attacher à penser ce qui se dit directement, métaphoriquement ou indirectement, par implicite, sur le temps dans le travail des historiens des Lumières, fussent-ils ou non philosophes. On distingue schématiquement quatre plans sur lesquels portent au XVIIIe siècle la réflexion et la pratique historiennes. La définition, le statut et l'utilisation du document historique d'abord. Ensuite, le sens du devenir historique, qu'on appelle, comme le fait Voltaire, sous le masque de l'abbé Bazin, la philosophie de l'Histoire. Ensuite, l'écriture du récit historique: ses modalités et ses règles. Enfin, la fonction de l'Histoire. De ces quatre axes fondamentaux, me semble-t-il, dérivent des questionnements ou des intérêts secondaires. Ainsi de la philosophie de l'Histoire, une réflexion sur l'événement pertinent permettant de juger du mouvement historique, de l'élargissement du champ de l'Histoire, de la définition d'une histoire culturelle, appelée encore 'histoire de l'esprit humain.' De la réflexion sur le document découlent la définition des conditions de possibilité de l'histoire elle-même comme reconstitution vraie du passé, avec pour 
affirmation essentielle le refus des fables et des mythologies fondatrices, le caractère privilégié, presque unique, du document écrit jusqu'aux grands chantiers archéologiques de la fin du siècle, les rapports entre Histoire et vérité. De la définition des règles de composition du récit historique se déduit la comparaison avec le récit romanesque qui débouche sur l'enjeu de vérité présent dans l'un et absent dans l'autre. Ce qui montre que ces divers axes ne correspondent pas à des questionnements spécifiques et à des cloisonnements stricts. De la réflexion sur la fonction de l'Histoire naît un débat sur la postérité, la morale sociale ou le recours à la mémoire collective.

Je ne prétends pas épuiser, par ce survol, tous les champs que parcourt la pratique historienne des Lumières ni même mettre au jour les relations qui les unissent, leurs chevauchements, leurs empiétements, leurs contradictions même qui composent cette structure mystérieuse que les historiens de l'Histoire tentent de décrire et d'explorer. Ce n'est pas mon propos ici, et j'ai déjà tenté partiellement de le faire dans mes travaux publiés sous le titre Le Règne de l'Histoire.

Si l'on continue à interroger la recherche dix-huitièmiste, on y constate un manque étonnant, jamais confessé. S'il existe des études sur l'Histoire, la narration, les ruines, il est rare qu'on s'attache à la conception, aux représentations du temps. On s'en tient pour toute analyse à des schémas assez convenus: continu ou discontinu, récit rétrospectif, va-etvient entre passé et présent, récit linéaire et cumulatif. On parle plus de diégétique, en tous ses états, que de temporalité romanesque. Le temps pourtant distendu et malléable du roman épistolaire attire peu souvent l'attention de ses commentateurs. La technique de la répétition, si souvent utilisée chez Sade, n'a jamais vraiment fait l'objet d'une analyse qui mette l'accent sur la dimension temporelle spécifique du temps et de la philosophie sadienne. Et pourtant le rapport du roman à l'histoire continue à interroger les consciences dix-huitièmistes.

Dans le discours critique, les rapports entre littérature et Histoire relèvent soit des conditions de production du texte littéraire, l'Histoire étant alors entendue comme une causalité, soit à l'inscription plus directe de l'histoire dans le texte, un peu dans la tradition du roman historique, soit enfin aux conditions historiques qui président à la réception du texte littéraire. Avant, dedans et après. Ce qui revient à dire que $l^{\prime}$ Histoire est ici prise en compte globalement sans que l'on s'interroge sur la représentation du temps qu'elle implique ou véhicule. Réduite à des forces sociales, à des institutions culturelles ou politiques, l'Histoire est à la limite comme atemporelle, coupée de son socle, dénuée de sens, ce qui, on me l'accordera, est quand même un paradoxe. 
$\mathrm{Au}$ terme de cette errance introductive, on tentera maintenant de repérer le travail dans la pratique littéraire du XVIIIe siècle, non de l'Histoire majestueuse et armée comme pour la parade, mais des représentations du temps : linéaire, cyclique, discontinu ou continu, régulier ou par à coups, délimitant des blocs événementiels à partir desquels se constitue son sens et que l'Histoire véhicule, met en scène ou illustre.

On sait que l'une des ruptures épistémologiquement majeures du XVIIIe siècle est le passage, ambigu, décalé d'une histoire cyclique (temps circulaire ramenant les choses à leur origine, parcours du néant des origines au néant de la destruction finale) à un temps linéaire, qui implique une histoire liée au progrès des formes politiques, de l'esprit humain, de la culture... Pas plus qu'une hirondelle ne fait le printemps, un temps nouveau ne chasse un temps ancien. Longtemps les deux représentations se sont mêlées et ont cohabité dans des articulations étranges et parfois jugées tératologiques. Chez Rousseau, l'histoire est d'abord immobile dans l'état de nature, puis linéaire et orientée contradictoirement ensuite, pour enfin entrer dans un cycle qui par l'aliénation des pouvoirs pervers, par le règne de l'inégalité absolue va réinscrire l'humanité dans une égalité du bien partagé qu'ils engendrent. Pour Voltaire, la vision linéaire du devenir se combine avec la théorie des quatre siècles, sommets de l'Histoire culturelle de l'humanité en devenir. Valable pour la seule Europe (et pour la Chine), ce schéma est inapplicable à l'Afrique et aux Samoyèdes ou aux Sarmates, dont le primitivisme arriéré, la violence régressive et toujours menaçante hante Voltaire. Si deux grandes représentations du temps historique se partagent grosso modo le XVIIIe siècle, il faut se méfier des simplifications abusives, non seulement dans l'ordre de leur succession mais dans leur différenciation même. La présence d'un texte comme Esquisse d'un tableau historique des progrès de l'esprit humain de Condorcet en pleine Révolution est trompeuse, car par son optimisme courageux en pleine tourmente, et alors que son auteur va périr sur l'échafaud, elle semble indiquer que la vision cumulative, positive du temps historique a définitivement triomphé. Or, tout prouve que l'angoisse des temps à venir, la crainte d'une néantisation qui proviendrait d'un temps ténébreux, à visage d'Apocalypse, sont bien présentes au delà des masques qui les occultent au regard de l'enquêteur. Citons pour mémoire les nostalgies diverses qui vont du Monde primitif de Court de Gébelin à la Philosophie de la nature de Delisle de Sales et aux remugles d'un rousseauisme affadi, dont participe Paul et Virginie. Pensons aussi au discours politique, comme le montre l'analyse menée ici même par Jean-Jacques Tatin. ${ }^{2}$ 
Il ne s'agit point de rechercher une transcription événementielle. Je l'ai fait, en mon temps, en analysant l'image du temps de la Révolution d'Angleterre dans Athalie, ou plus largement dans la tragédie classique jusqu'en 1715. Ici il faut traquer une représentation, un imaginaire du temps de l'Histoire qui ordonnent sa structure, le déroulement narratif de la durée et du devenir. Remarquons qu'en ce qui regarde l'écriture, le passage d'une représentation à une autre entraîne des mutations métaphoriques qu'on n'a pas suffisamment, jusqu'à une date récente, prises en compte. La représentation cyclique du temps qui unit le domaine du temps religieux à celui du temps laïque trouve à s'exprimer métaphoriquement dans le destin humain: néant, naissance, enfance, force de l'âge adulte, déclin et mort. On en trouve trace chez tous les penseurs politiques: de Jean Borin à Hobbes et à Bossuet. Le passage à une vision linéaire de l'histoire devrait rendre cette métaphore caduque. De même que la métaphore des fleuves allant se perdre dans la mer. Non sans hésiter, on lui substitua, d'abord fragmentairement, puis avec plus d'amplitude, la métaphore de la marche d'une troupe ou d'une caravane, que Lamartine dans Jocelyn exploitera à l'excès. Diderot, comme l'a montré Jean-Jacques Tatin, invente une métaphore intermédiaire à double signification, qui unit montagne (ou hauteur) et précipice, stabilité et chute: ${ }^{3}$

[...] il a fallu des siècles pour amener notre instant fatal; et cet instant pouvait être retardé par des lois et des institutions sages, si nous en avions eu. Songez, madame [il s'agit de l'Impératrice Catherine] que je vous présente l'éboulement d'un grand amas de grains de sable que des circonstances fortuites avaient entassés, au lieu qu'il dépend de Votre Majesté de placer la base de votre pyramide sur le roc et d'en lier les différentes parties par des crampons de fer. Le roc s'affaisse, il est vrai, les pierres se disjoignent, et l'édifice s'écroule à la longue; mais il a duré cent siècles; cent siècles d'un bonheur continu et procuré par les travaux et le génie étonnant de Votre Majesté à trente millions d'hommes, ne suffiront-ils pas son âme vaste et grande? (Mémoires à Catherine II) ${ }^{4}$

Un peu avant ce sommet de la philosophie, la Querelle des Anciens et des Modernes avait constitué un laboratoire d'écriture métaphorique du temps culturel. Je n'ai pas mené avec soin cette enquête sur les métaphores du temps, mais il me semble qu'il serait d'un intérêt majeur de repérer tout une constellation métaphorique en mutation, à articulations multiples, autour par exemple du surgissement de la lumière, travaillant l'image du soleil levant puis s'abîmant, pour céder la place aux ténèbres, dans la mer. Sur des imaginaires métaphoriques de base comme la marche ou l'escalade, le destin du corps humain, le courant de l'eau, le 
dépérissement de la nature, la contemplation méditative des ruines parviennent à s'exprimer des représentations du temps historique.

On a souvent souligné qu'au roman linéaire, aussi volumineux fût-il, mais simple dans sa structure temporelle, en tenant pour peu les histoires intercalées, la fin du XVIIe siècle commence à substituer de nouvelles structures narratives: le roman à la première personne d'une part et d'autre part le roman épistolaire dans sa forme la plus épurée entendons ici Les lettres de la religieuse portugaise. Je voudrais essayer de comprendre quelles relations unissent leurs structures à ces représentations du temps historique que j'ai évoquées précédemment. Remarquons d'abord que le récit à la première personne, rétroactif, est semblable au discours historique qui part des origines pour procéder à une avancée dans le temps. A cette différence que le narrateur dans le roman est sujet de l'énoncé et de l'énonciation alors que toute la pratique historique tend de plus en plus à distancier l'historien de sa narration de l'histoire. Pierre Bayle dans le Dictionnaire historique et critique ira jusqu'à théoriser cette distance à travers la figure de Melchisédech, grand prêtre d'Israël, dont les Textes Sacrés affirment qu'il n'avait ni ascendance ni descendance, ce qui signifie une étrangeté radicale aux autres, à ses engagements terrestres, aux siens, à sa famille, à ses fidélités religieuses ou citoyennes, qui doit être celle de l'historien même. Le récit à la première personne - et ce n'est pas un coup de hasard s'il emprunte un de ses titres les plus fréquents à la panoplie historique en utilisant le terme 'mémoires' - est un récit cumulatif, récit d'une trajectoire par épisodes successifs, à la façon de la novela picaresca. La représentation du temps qui lui est sous-jacente, dans la majorité des cas, conduit le héros des bas-fonds de la misère ou du néant social à une position enviable dans la société. Pensons au Paysan parvenu, qui, malgré son inachèvement, est dans cette perspective exemplaire. Cette vision positive du temps dans le roman (valeur et structure) devient majoritaire quand elle parvient à se formuler dans les domaines esthétique et scientifique à travers la Querelle des Anciens et des Modernes, où se modifie en profondeur une vision du temps jusqu'ici largement entachée de théologie. Le passage d'un roman tragique (les histoires tragiques du XVIe et du XVIIe siècles) à ce roman d'apprentissage social comme le Gil Blas de Santillane est révélateur d'une mutation des représentations du temps historique. L'analyse systématique des dénouements (tragiques ou optimistes), leur distribution dans le temps montrerait les ruptures et les hésitations. A cet égard, on notera que la montée des dénouements tragiques avec la fin du siècle (Les Liaisons dangereuses, Paul et Virginie, les romans de Sade) réintroduisent en plein optimisme officiel une dimension tragique et noire du temps qu'on croyait oubliée. 
Sans tenir compte de la nature des dénouements dans l'analyse du roman épistolaire, on peut, au demeurant, remarquer que l'argument traditionnel qui les fonde en vraisemblance fait de l'éditeur un peu plus qu'un scribe, comme on le prétend ordinairement. Le manuscrit, les lettres trouvées ou transmises ressemblent à s'y méprendre aux documents dont dispose l'historien. On a là du Mabillon face à l'épistolaire. On réunit, on classe, on constate les manques, comme le faisait le scrupuleux bénédictin en définissant les règles de sa diplomatique. L'articulation du roman au récit historique qui fascine tant le XVIIIe siècle, pensons à Lenglet-Dufresnoy (De l'histoire justifiée contre les romans, 1734) trouve ici une nouvelle forme plus intéressante que le débat un peu vain sur la morale du roman ou même sur sa vérité. C'est en ces termes de position qu'il convient, me semble-t-il, de repenser pour une large part la volonté de crédibilité que manifeste le roman durant tout le XVIIIle siècle à travers la fable du manuscrit trouvé, et du romancier simple éditeur. L'installation du romancier en historien archiviste illustre la valeur de modèle qu'est en train d'acquérir l'Histoire.

A bien y regarder, l'histoire du roman à la première personne utilise le schéma fondamental des philosophies cumulatives et linéaires du devenir historique. L'historien de soi, entendons l'auteur d'autobiographie, organise sa démarche en cherchant d'abord un temps fragmenté des origines, dans lequel trouve à s'inscrire le programme d'une vie. On recommande à l'historien de rechercher une suite d'événements fondateurs ou signifiants - comme le veut le programme tracé par Fénelon dans sa Lettre à l'Académie - et ainsi s'écrivent ces histoires des révolutions qui se publient tout au long du siècle. Cette position est semblable à celle de l'autobiographe qui choisit d'expliquer ce qu'il est par des événements fondateurs ou révélateurs de l'intime et du caché. Mais il doit aussi décrire un flux, un déploiement, qui sont le récit même en continu de sa vie. C'est cette dualité qui permet de saisir l'articulation des deux premiers livres des Confessions aux livres suivants et de comprendre leur organisation en boucles récurrentes, selon un temps régressif, qui fait que la 'dixième promenade' s'organise narrativement du présent au passé et non du passé au présent.

Dans un souci de crédibilité, à rattacher à des modèles culturels, l'écrivain se veut tout autant archiviste qu'historien, ou plutôt mêle les deux figures selon un tissage complexe qui prouve que le temps de l'Histoire, tel qu'il se pense, est à l'oeuvre dans la trame narrative elle-même.

La pratique de l'Histoire, si importante pour les Lumières, n'est pas à dissocier, comme on serait naïvement tenté de le faire, de la pratique plus directement militante des philosophes. Ainsi, on ne peut ignorer les représentations du temps et de l'Histoire qui légitiment, en dernière 
instance, l'entreprise encyclopédique. Il faut refuser l'interprétation triomphaliste, largement acceptée, qui voit à l'œuvre dans l'Encyclopédie un imaginaire du temps essentiellement cumulatif, qui aboutirait à une geste de prise de possession du monde, illustrant avec orgueil l'ascension bourgeoise. Elle représente, en réalité, à bien des égards, un symptôme de fragilité d'un monde culturel, politique et social menacé. Dans cette perspective, il faut relire l'article 'Encyclopédie' lui-même: '[q]ue $l^{\prime}$ Encyclopédie devienne un sanctuaire [écrit Diderot] où les connaissances des hommes soient à l'abri des temps et des révolutions.'

On voit bien comment le projet encyclopédique s'inscrit résolument dans une vision catastrophique des temps à venir, et représente, de fait, une opération de sauvetage. Dans cette optique, elle est essentiellement pensée comme un inventaire, écrit et imagé, à partir duquel pourra se reconstruire, dans un paysage de ruines, le monde détruit. L'espoir n'est donc pas placé dans un temps producteur, mais dans un livre conçu comme une sorte de mémoire testamentaire. Une utopie comme celle de Grivel, L'île inconnue, montre la justesse de cette hypothèse.

Il convient donc de reconnaître, me semble-t-il, à partir de la visée encyclopédique un double ancrage de la pratique philosophique. Elle est souvent d'intervention ponctuelle, limitée et elle fait qu'à travers le débat polémique s'instaure un temps discontinu. La querelle autour des Philosophes de Palissot ou de l'Affaire des Cacouacs, ou la réaction de Jean-Jacques Rousseau aux condamnations qui frappent son œuvre, illustrent bien ce modèle d'écriture, temporalisé à l'extrême et pourtant détemporalisé, si on le pense dans la longue durée.

L'inscription des œuvres de Voltaire dans cet équilibre fragile du temporaire et de l'oubli est exemplaire. Aucun enjeu de durée pour lui dans les contes, les épigrammes, les lettres, les mille interventions en faveur des Calas, des Sirven, des Montbailli ou du chevalier de La Barre. Le temps culturel n'a d'épaisseur et de durée qu'au regard de l'épopée, des tragédies et de l'œuvre historique. Pour Diderot lui-même, la pratique philosophique entretient des relations complexes avec le temps. Les représentations acquises sont soumises par lui à un incessant travail métaphorique pour parvenir à exprimer une vision catastrophique dans les textes qu'il publie, mais aussi un acte d'espérance dans l'attente d'aubes nouvelles qu'expriment tous ces manuscrits qu'il garde en portefeuille et qui représentent paradoxalement un pari optimiste sur un temps cumulatif de libération et de progrès, en opposition évidente avec une réelle désespérance du présent. La palette est infinie. Tirons-en une leçon: cessons de voir la philosophie des Lumières à l'aune du progrès, dont Voltaire serait le représentant le plus exemplaire. 
Il reste à proposer quelques hypothèses d'enquête concernant le théâtre. On s'est longtemps étonné que la tragédie ait pu survivre dans l'environnement des Lumières et que les plus grands succès d'alors aient été les tragédies de Voltaire ou Le siège de Calais de Belloy. On s'interroge, perplexe, sur ce que l'on juge une incohérence et sur la cohabitation d'un monde figé, codifié et d'un mode d'intervention souple, actif, inventif et libre. Au poids des codifications esthétiques, à la force de l'éducation reçue, du goût classique qui est commun à tous - LUI et MOI ne se mettent-ils pas d'accord, toutes affaires cessant, pour reconnaître le génie incontestable de Jean Racine? - à ces raisons donc, il faut ajouter l'imaginaire du temps que véhicule l'univers tragique, et qui hante la conscience des hommes du siècle. La tragédie, même lorsqu'elle modernise son sujet, demeure le temps du destin et de la finitude. Dans un monde qui se laïcise, où l'on exalte, comme dans Le Mondain de Voltaire, les bonheurs de ce siècle de fer, où l'on croit globalement à une marche assurée de l'histoire des progrès de l'esprit humain, la tragédie rappelle les menaces présentes comme des vigies aux horizons de l'Histoire, le temps comme crise, dont la résolution sera la mort. Toute tragédie constitue une catastrophe exhibée, donnée en exemple, comme pour rappeler la vérité trop vite oubliée du néant et des passions destructrices.

Outre les analyses et les questionnements dont le drame bourgeois est justiciable, il est urgent d'analyser le travail auquel y sont soumises les représentations du temps. Une remarque de Beaumarchais dans L'essai sur le genre dramatique sérieux de 1767 peut nous y aider. Elle est peu et mal commentée, considérée comme une boutade, alors qu'elle représente un moyen de réinscrire le drame dans les tensions qui traversent l'imaginaire du temps. 'Que me font à moi, écrit Beaumarchais, sujet d'un État monarchique du XVIIIe siècle, les révolutions d'Athènes et de Rome?' Une telle affirmation niant l'utilité d'un type d'Histoire encore à la mode (Les Histoires des Révolutions) souligne une coupure entre deux attentes, deux modes d'explication du devenir historique, deux temps: une histoire de troubles, de changements et une histoire immobile, atone, qui pourrait être celle advenue au XVIIIe siècle. Tout ici se confond. L'impossibilité de la leçon par l'Antique pour le temps présent est le signe d'une hétérogénéité des philosophies du devenir et des histoires spécifiques, marquant le refus d'une histoire, jusqu'ici explicative de toute histoire. On sort du cycle antique pour prendre une autre mesure du temps, comme si par là se signifiait l'épuisement d'une représentation. Le temps historique est senti comme modulable. Si le théâtre devient celui des conditions, des familles, des marchands - je renvoie à ce que peuvent en dire Louis-Sébastien Mercier dans L'examen de la tragédie française, et Diderot dans Les entretiens sur le fils naturel c'est parce que le monde contemporain, le commerce, la circulation des 
marchandises, les crises de l'intime sont devenus les temps forts de $l^{\prime}$ Histoire au regard de ceux qui pensent son devenir.

L'exclusion du politique - le célèbre 'la tragédie ce sont les rois' est à prendre en compte parce que l'Histoire nouvelle, cette histoire des moeurs selon Voltaire, l'excluait déjà. Il y a rencontre entre une figuration théâtrale et un nouvel objet du discours historique. Si l'on pousse l'interrogation plus avant, si l'on tente de rattacher l'utilisation théâtrale des conditions aux valorisations politiques qui sont liées au commerce, facteur de développement des libertés, on en vient tout naturellement à faire de cette histoire des temps présents l'image d'un temps immobile ou cumulatif au-delà des crises apparemment limitées à l'intimité des familles.

On oublie fréquemment de rappeler une des fictions qui est utilisée dans Le fils naturel. Diderot serait entré en possession d'un manuscrit rédigé par Dorval (le héros de la pièce), à partir d'une aventure réelle, sur l'injonction de son père pour être représentée d'âge en âge par sa descendance, et prolonger ainsi la mémoire d'une des circonstances les plus importantes de sa vie et de la conduite $q^{\prime} \mathrm{u}^{\prime}$ tient chacun des membres de la famille qui s'y trouvait engagé. Diderot, caché, aurait assisté à une de ces représentations privées. Ce qui dévoile l'imaginaire du spectateur-voyeur, mais aussi un rituel de la commémoration des origines. On a bien ici un temps répété, encore qu'inscrit dans une problématique de la reprise, divergente de celle du cycle traditionnel.

Pour en finir, je voudrais tenter de rapprocher d'une manière plus hypothétique encore certaines des techniques nouvelles mises en œuvre dans le drame bourgeois des rapports au temps qu'entretient l'imaginaire des Lumières. Le refus du vers au théâtre rejoint la volonté d'inventer une forme nouvelle du récit historique plus proche de la réalité, plus en prise sur l'époque qui fait que l'on élimine portraits et harangues, ainsi que le recommande Voltaire. L'écriture historique, comme on le voit dans l'Essai sur les moeurs, prend en charge un double registre: temps heurté et dramatisé de la crise, temps pédagogique de l'anecdotique, temps ramassé de l'événementiel et temps suspendu de la synthèse. L'écriture théâtrale en est proche, qui utilise tableau, pantomime et monologue d'une part, expression d'un temps immobile et, d'autre part, l'art de la réplique, l'écriture de la péripétie, qui renvoient à un temps accéléré et discontinu.

Je ne vais pas pousser plus avant cette analyse aventureuse des homologies et des recoupements. Quelle que soit la validité des hypothèses ici avancées et des analyses esquissées, elles ont, à mes yeux, le mérite de rappeler que l'imaginaire du temps travaille et légitime, à parts 
égales, le récit de reconstruction des ombres de l'historien et la pratique inventive du romancier ou du dramaturge.

JEAN M. GOULEMOT

Université de Tours, Institut Universitaire de France

\section{Notes}

1 Voir, à ce sujet, mon ouvrage Le règne de l'histoire (Paris: Albin Michel, 1997).

2 Voir 'Les résurgences des représentations catastrophiques de l'histoire à la fin du XVIIIe siècle.'

3 Ibid.

4 Diderot, Mélanges à Catherine II, dans CEuvres complétes (Paris: Robert Laffont, 1994) III, 217. 